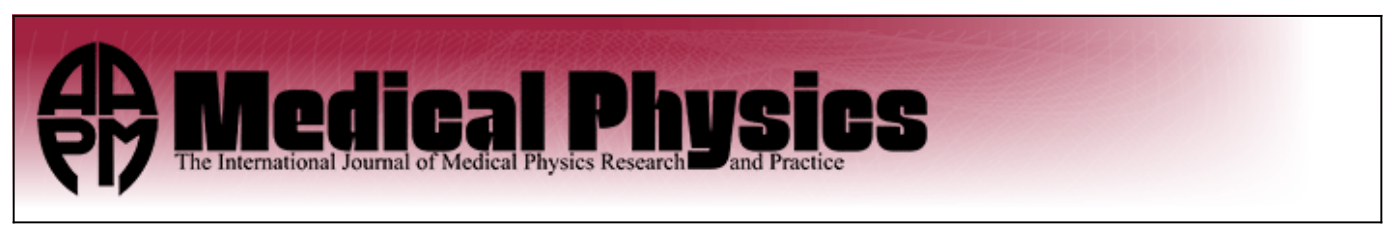

\title{
Alpha image reconstruction (AIR): A new iterative CT image reconstruction approach using voxel-wise alpha blending
}

Christian Hofmann, Stefan Sawall, Michael Knaup, and Marc Kachelrieß

Citation: Medical Physics 41, 061914 (2014); doi: 10.1118/1.4875975

View online: http://dx.doi.org/10.1118/1.4875975

View Table of Contents: http://scitation.aip.org/content/aapm/journal/medphys/41/6?ver=pdfcov

Published by the American Association of Physicists in Medicine

\section{Articles you may be interested in}

Combined iterative reconstruction and image-domain decomposition for dual energy CT using total-variation regularization

Med. Phys. 41, 051909 (2014); 10.1118/1.4870375

Iterative image-domain decomposition for dual-energy CT

Med. Phys. 41, 041901 (2014); 10.1118/1.4866386

Radiation dose reduction in medical x-ray CT via Fourier-based iterative reconstruction

Med. Phys. 40, 031914 (2013); 10.1118/1.4791644

Characterization of adaptive statistical iterative reconstruction algorithm for dose reduction in CT: A pediatric oncology perspective

Med. Phys. 39, 5520 (2012); 10.1118/1.4745563

Iterative image reconstruction for CBCT using edge-preserving prior

Med. Phys. 36, 252 (2009); 10.1118/1.3036112

\section{SIVARTER, FASTER

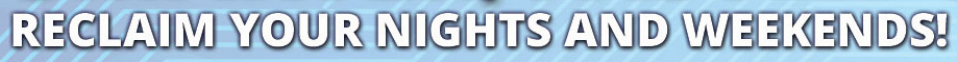

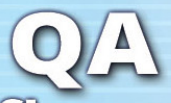

One minute IMRT and VMAT QA!

no arrays, chambers, film or EPID necessary 


\title{
Alpha image reconstruction (AIR): A new iterative CT image reconstruction approach using voxel-wise alpha blending
}

\author{
Christian Hofmann \\ Institute of Medical Physics, Friedrich-Alexander University (FAU), Erlangen 91052, Germany
}

Stefan Sawall and Michael Knaup

Medical Physics in Radiology, German Cancer Research Center (DKFZ), Heidelberg 69120, Germany

Marc Kachelrie $\beta^{\text {a) }}$

Institute of Medical Physics, Friedrich-Alexander University (FAU), Erlangen 91052, Germany and Medical Physics in Radiology, German Cancer Research Center (DKFZ), Heidelberg 69120, Germany

(Received 24 January 2014; revised 26 March 2014; accepted for publication 27 April 2014; published 28 May 2014)

Purpose: Iterative image reconstruction gains more and more interest in clinical routine, as it promises to reduce image noise (and thereby patient dose), to reduce artifacts, or to improve spatial resolution. Among vendors and researchers, however, there is no consensus of how to best achieve these aims. The general approach is to incorporate a priori knowledge into iterative image reconstruction, for example, by adding additional constraints to the cost function, which penalize variations between neighboring voxels. However, this approach to regularization in general poses a resolution noise trade-off because the stronger the regularization, and thus the noise reduction, the stronger the loss of spatial resolution and thus loss of anatomical detail. The authors propose a method which tries to improve this trade-off. The proposed reconstruction algorithm is called alpha image reconstruction (AIR). One starts with generating basis images, which emphasize certain desired image properties, like high resolution or low noise. The AIR algorithm reconstructs voxel-specific weighting coefficients that are applied to combine the basis images. By combining the desired properties of each basis image, one can generate an image with lower noise and maintained high contrast resolution thus improving the resolution noise trade-off.

Methods: All simulations and reconstructions are performed in native fan-beam geometry. A water phantom with resolution bar patterns and low contrast disks is simulated. A filtered backprojection (FBP) reconstruction with a Ram-Lak kernel is used as a reference reconstruction. The results of AIR are compared against the FBP results and against a penalized weighted least squares reconstruction which uses total variation as regularization. The simulations are based on the geometry of the Siemens Somatom Definition Flash scanner. To quantitatively assess image quality, the authors analyze line profiles through resolution patterns to define a contrast factor for contrast-resolution plots. Furthermore, the authors calculate the contrast-to-noise ratio with the low contrast disks and the authors compare the agreement of the reconstructions with the ground truth by calculating the normalized cross-correlation and the root-mean-square deviation. To evaluate the clinical performance of the proposed method, the authors reconstruct patient data acquired with a Somatom Definition Flash dual source CT scanner (Siemens Healthcare, Forchheim, Germany).

Results: The results of the simulation study show that among the compared algorithms AIR achieves the highest resolution and the highest agreement with the ground truth. Compared to the reference FBP reconstruction AIR is able to reduce the relative pixel noise by up to $50 \%$ and at the same time achieve a higher resolution by maintaining the edge information from the basis images. These results can be confirmed with the patient data.

Conclusions: To evaluate the AIR algorithm simulated and measured patient data of a state-of-the-art clinical CT system were processed. It is shown, that generating CT images through the reconstruction of weighting coefficients has the potential to improve the resolution noise trade-off and thus to improve the dose usage in clinical CT. (C) 2014 American Association of Physicists in Medicine. [http://dx.doi.org/10.1118/1.4875975]

Key words: Key words: iterative reconstruction, regularization, clinical-CT

\section{INTRODUCTION}

In the recent years, significant efforts have been done to develop new iterative image reconstruction algorithms, which outperform standard filtered backprojection (FBP) methods with the aim to lower the noise and improve spatial resolution properties. The underlying motivation is to further reduce patient dose ${ }^{1}$ and to reduce artifacts, for example, blooming artifacts in cardiac CT, which arise from high contrast calcification structures due to the limited spatial resolution. ${ }^{2-5}$ 
Today, all CT vendors offer iterative reconstruction approaches in their products. However, these methods are still in the phase of clinical evaluation, but results are very promising. ${ }^{6-10}$ There are several recent review papers which compare the vendor implementations of iterative reconstructions in clinical CT among each other. ${ }^{11-13}$ It turns out, that among these approaches rawdata-based iterative reconstruction methods have shown most promising results. ${ }^{7,9}$ Iterative reconstruction algorithms offer the possibility to incorporate nonidealities in the problem description: Statistical algorithms are based on a noise model and thus consider the photon statistics. ${ }^{14,15}$ Prior knowledge such as positivity or smoothness can be considered in an iterative algorithm by regularization approaches. ${ }^{16-19}$ Vendors and researchers incorporate this a priori knowledge into the reconstruction process by adding additional constraints to the objective function of the iterative reconstruction algorithm, which penalize variations between neighboring voxels. Many penalty terms have been proposed like q-generalized Gaussian Markov random fields, ${ }^{20}$ total variation (TV), and Huber penalty, ${ }^{16,19,21-23}$ or other anisotropic filters. ${ }^{18,24}$ In general, these regularization approaches pose a resolution noise trade-off. Regularized iterative reconstruction often fails at the resolution limit or when the contrast of details is in the range of the noise level. It can be a challenging task to find robust parameter settings. The higher the dimensionality of the parameter space, the more complicated is it to find parameter settings suitable to generate stable and predictable results without altering anatomical information. Driven by the desire to combine the favored image properties of regularization results with various parameter settings to overcome this trade-off, we propose a new empirical method to incorporate regularization in iterative reconstruction. The proposed algorithm is called alpha image reconstruction (AIR). It is based on our recent work presented in Ref. 25. The idea is to generate basis images which emphasize certain image properties like high resolution, high low contrast detectability, or low noise. These images could be regularized reconstructions, postreconstruction filtered images, or reference reconstructions like FBP, for instance. Regularization is incorporated into the reconstruction by the basis images. The AIR algorithm reconstructs voxel-specific weighting coefficients that are applied to combine the basis images. By combining the desired properties of each basis image, one can generate an image with lower noise and maintain high contrast resolution, thus improving the resolution noise tradeoff.

The paper is arranged as follows: In Secs. 2.A and 2.B, we derive the proposed iterative reconstruction algorithm. Sections 2.C-2.F summarize details about the simulation and the quantitative analysis. In Sec. 3.A, we present results for the simulated phantom and in Sec. 3.B we present clinical data.

\section{MATERIALS AND METHODS}

\section{A. Statistical image reconstruction}

Before introducing the AIR algorithm, we want to give a short recapitulation of iterative statistical reconstruction based on the maximum a posteriori (MAP) approach, as we want to compare the proposed method against a state of the art iterative reconstruction algorithm. The statistical reconstruction algorithms used throughout this paper are based on the Poisson model for photon intensities. The conditional likelihood $P(\boldsymbol{p} \mid \boldsymbol{f})$ can be derived for the acquired projection data $\boldsymbol{p}$ and the unknown image $\boldsymbol{f}$, which is to be calculated. The rule of Bayes' gives an expression for the conditional likelihood

$$
P(\boldsymbol{f} \mid \boldsymbol{p})=\frac{P(\boldsymbol{p} \mid \boldsymbol{f}) P(\boldsymbol{f})}{P(\boldsymbol{p})} .
$$

With the Bayesian framework the reconstruction problem may be formulated as MAP estimate

$$
\boldsymbol{f}=\arg \max _{\boldsymbol{f}} \log P(\boldsymbol{p} \mid \boldsymbol{f})+\log P(\boldsymbol{f}),
$$

after dropping the term independent of $f$ and taking the logarithm in Eq. (1). The first term on the right-hand side in Eq. (2) is called the log-likelihood. A good approximation to $\log P(\boldsymbol{p} \mid \boldsymbol{f})$ can be achieved by a second-order Taylor expansion. ${ }^{26}$ Applying this approximation to Eq. (2) yields

$$
\begin{aligned}
\boldsymbol{f} & =\arg \min _{\boldsymbol{f}}(\boldsymbol{X} \cdot \boldsymbol{f}-\boldsymbol{p})^{T} \cdot \boldsymbol{W} \cdot(\boldsymbol{X} \cdot \boldsymbol{f}-\boldsymbol{p})+\eta U(\boldsymbol{f}) \\
& =\arg \min _{\boldsymbol{f}}\|\boldsymbol{X} \cdot \boldsymbol{f}-\boldsymbol{p}\|_{\boldsymbol{W}}^{2}+\eta U(\boldsymbol{f}),
\end{aligned}
$$

where $\boldsymbol{X}$ is the forward projection (x-ray transform). $\boldsymbol{W}$ is a diagonal matrix with statistical weights, $U(\boldsymbol{f})$ is a regularization term which is equal to $\log P(f)$ except for an additive constant, and $\eta$ is a trade-off parameter. For transmission tomography, the diagonal elements of $\boldsymbol{W}$ are proportional to the detector counts. ${ }^{20}$ They represent the credibility of the data. This means, that the more quanta that are measured, the smaller the signal variance and consequently the stronger the data are weighted as reliable within the optimization. Forward and backprojection are performed in the native fanbeam geometry. As forward projection operation we use the Joseph forward projector ${ }^{27}$ and as backprojection operation we use a destination-driven backprojection algorithm, i.e., we did voxel-driven backprojection with bilinear interpolation on the detector. Using $\eta=0$ means that no regularization term is used and the resulting reconstruction is called weighted least squares (WLS) solution. It is well known, that unregularized statistical iterative reconstructions, like the WLS, tend to converge to excessively sharp and noisy images. ${ }^{18}$ Using $\eta>0$ means that we incorporate further knowledge in form of a penalty term. Thus, the WLS solution becomes a penalized WLS (PWLS) solution. We compare AIR against the PWLS reconstruction with TV regularization, as TV has gained much attention in the recent years and is well known. ${ }^{19}$ Furthermore, it is easy to implement and has only view parameters, making it easy to comprehend the results. With the TV regularization the optimization problem becomes

$$
\boldsymbol{f}=\arg \min _{\boldsymbol{f}}\|\boldsymbol{X} \cdot \boldsymbol{f}-\boldsymbol{p}\|_{\boldsymbol{W}}^{2}+\eta T V(\boldsymbol{f}) .
$$


The total variation cost penalty is defined as

$$
T V(\boldsymbol{f})=\sum_{x=1}^{N_{x}-1} \sum_{y=1}^{N_{y}-1} \sqrt{(f(x+1, y)-f(x, y))^{2}+(f(x, y+1)-f(x, y))^{2}+\epsilon},
$$

where the parameter $\epsilon$ is a small positive constant to avoid discontinuities. We denote the PWLS with TV penalty PWLSTV.

\section{B. Proposed method: AIR}

The AIR algorithm basically is a different approach to regularization and thus to incorporate prior knowledge into iterative reconstruction to improve the resolution noise trade-off. One starts with reconstructing basis images $\boldsymbol{f}_{b}$, which could be regularized reconstructions, postreconstruction filtered images, or FBP, for instance. The number of basis images is denoted with $B$. Through these basis images prior knowledge (regularization) is incorporated into the reconstruction process. AIR reconstructs voxel-specific weighting coefficients $\boldsymbol{\alpha}_{b}$ for each basis image $\boldsymbol{f}_{b}$ that are applied to combine the basis images, finding the combination which produces the maximal rawdata fidelity. Thus, each basis image on its own may suffer from certain deficiencies, due to too strong regularization, for instance. Thus, the algorithm aims at combining the favored properties of the basis images like high resolution and low noise. This is done by minimizing the following cost function:

$$
\begin{aligned}
\boldsymbol{\alpha} & =\left(\begin{array}{c}
\boldsymbol{\alpha}_{1} \\
\boldsymbol{\alpha}_{2} \\
\vdots \\
\boldsymbol{\alpha}_{B}
\end{array}\right)=\arg \min _{\boldsymbol{\alpha}} C(\boldsymbol{\alpha}) \\
& =\arg \min _{\boldsymbol{\alpha}}\left\|\boldsymbol{X} \cdot\left(\sum_{b=1}^{B} \boldsymbol{\alpha}_{b} \circ \boldsymbol{f}_{b}\right)-\boldsymbol{p}\right\|_{\boldsymbol{W}}^{2}+U(\boldsymbol{\alpha}),
\end{aligned}
$$

where $\boldsymbol{X}$ is the $\mathrm{X}$-ray transform, $\boldsymbol{p}$ is the rawdata vector, and $\boldsymbol{W}$ are the statistical weights as described in Sec. 2.A. The $\boldsymbol{f}_{b}$ are the basis images and the $\boldsymbol{\alpha}_{b}$ are the corresponding weighting images. The vector $\boldsymbol{\alpha}$ contains all weighting images. The expression $\boldsymbol{\alpha} \circ \boldsymbol{f}$ stands for the Hadamard product, i.e., the vectors are multiplied element wise. One has to determine the $\boldsymbol{\alpha}$-images by minimizing the above cost function. The result image is defined as

$$
\boldsymbol{f}_{\mathrm{AIR}}=\sum_{b=1}^{B} \boldsymbol{\alpha}_{b} \circ \boldsymbol{f}_{b}
$$

Note that $\sum_{b} \boldsymbol{\alpha}_{b} \neq 1$ in general. The regularization term $U(\boldsymbol{\alpha})$ is used to set certain constraints to the weighting images $\boldsymbol{\alpha}_{b}$ such as continuity. We define our penalty term $U(\boldsymbol{\alpha})$ as

$$
U(\boldsymbol{\alpha})=\beta \sum_{b=1}^{B} T V\left(\boldsymbol{\alpha}_{b}\right)+\gamma \sum_{b=1}^{B}\left\|\boldsymbol{\alpha}_{b}-\boldsymbol{d}_{b}\right\|_{2}^{2},
$$

where the vectors $\boldsymbol{d}_{b}$ are constant vectors. Here, we chose $\boldsymbol{d}_{b}=d_{b} \mathbf{1}$ with $d_{b}$ being a constant scalar value. In other words, all entries of $\boldsymbol{d}_{b}$ are identical and chosen to be $d_{b}$. In general, there may be other reasonable or even better ways to choose the entries of $\boldsymbol{d}_{b}$. This, however, is beyond the scope of this work. The TV penalty is used, defined in Eq. (6), to enforce homogeneity and to suppress the occurrence of noise in the weighting images. We use the TV penalty, because it has only few open parameters that can be tuned rather easily. Note that instead of using TV one could think of applying other penalties. The strength of the TV penalty is controlled by parameter $\beta$. The second penalty term defines the default value $\boldsymbol{d}_{b}$ of basis image $b$. Regarding this penalty term in Eq. (9) one can see that the minimum norm of zero is achieved when all values in the weighting images $\boldsymbol{\alpha}_{b}$ are equal to $\boldsymbol{d}_{b}$. In this paper, we use $\boldsymbol{d}_{b}=d_{b} \mathbf{1}$ with $d_{b}=1 / B$ such that the basis images default to an average value of $1 / B$. Thus, homogeneous regions in the image tend to be averaged. This is because homogeneous regions do not contribute much to the rawdata fidelity, apart from the mean value picked up by a ray segment running through such a region. As this penalty term is strictly convex, the overall cost function is strictly convex, too, and a unique global minimum exists. In the following, we will refer to regions in the basis images where the detail information is close to the correct image (i.e., the ground truth) as regions with a high correlation with the rawdata, due to the fact that the closer the reconstructed image to the ground truth, the higher is the rawdata fidelity. If regions strongly differ from the ground truth we refer to them as regions with a low correlation. By minimizing the overall cost function one finds the optimal voxel-wise combination of the basis images with respect to the rawdata fidelity and the penalty term $U(\boldsymbol{\alpha})$. The minimum norm of this cost function is reached when the weighting images will have large contributions in regions of the corresponding basis image which highly agree with the rawdata and low weighting otherwise because the smoothness constraint defined by the TV penalty prohibits arbitrary values in the weighting images. Thus, the highest contribution to a specific detail in the final result will be provided by the basis image best representing the real anatomical information in this specific region. In Secs. 3.A.1-3.A.3, we give a more detailed insight of how the method works in principle.

We minimize Eq. (7) with a gradient descent approach. For that we have to calculate the gradient of the cost function $C(\boldsymbol{\alpha})$. The gradient of Eq. (7) is

$$
\nabla C(\alpha)=\left(\begin{array}{c}
f_{1} \circ g \\
f_{2} \circ g \\
\vdots \\
f_{B} \circ g
\end{array}\right)+\nabla U(\alpha)
$$


with

$$
\boldsymbol{g}=2 \boldsymbol{X}^{T} \cdot\left(\boldsymbol{W} \cdot \boldsymbol{X} \cdot\left(\sum_{b=1}^{B} \boldsymbol{\alpha}_{b} \circ \boldsymbol{f}_{b}\right)-\boldsymbol{p}\right)
$$

$\boldsymbol{X}^{T}$ is the transpose X-ray transform and $\nabla U(\boldsymbol{\alpha})$ is the gradient of the penalty term for $\alpha$. One gradient descent update step for the weighting images can be written as

$$
\boldsymbol{\alpha}^{\nu+1}=\boldsymbol{\alpha}^{\nu}-\lambda \nabla C\left(\boldsymbol{\alpha}^{\nu}\right)
$$

where $v$ denotes the iteration step and $\lambda$ denotes the step length of the gradient descent method. The step length $\lambda$ was calculated by a backtracking line search method. ${ }^{28}$ To be sure that the gradient based minimization process converges to an unique global minimum, one has to ensure that the overall cost function is strictly convex. The rawdata fidelity term of cost function (7) is convex but underdetermined due to the weighted sum of the basis images. Thus, the rawdata fidelity term is convex but not strictly convex. The global minimum is not unique. The sum over the TV of the weighting images is convex but not strictly convex because the minimum norm can be achieved with an infinite number of solutions for $\boldsymbol{\alpha}$. Thus, the combined cost of the rawdata fidelity and the total variation penalty is convex but not necessarily strictly convex. However, the additional penalty term is a strictly convex function, as its minimal value can only be achieved when all $\boldsymbol{\alpha}_{b}$ equal $d_{b}$. Thus, the overall cost function is strictly convex and there is a unique global minimum and the overall cost function is suited for minimization by a gradient descent approach. We initialize $\boldsymbol{\alpha}$ with zero. Regions outside the phantom or patient are clipped to zero in the corresponding weighting images after reconstruction. This is done by thresholding. We do so for cosmetic reasons because then one can see the boundaries of the object. All iterative algorithms were iterated until convergence. Defining convergence in practice can be difficult. To be sure that all algorithms reached convergence, we analyzed the cost value as function of the iteration number and did extensive visual inspection of succeeding iterations. We found that 500 iterations are sufficient for all algorithms to converge. In order to be consistent, this number of iterations was used for all reconstructions. We did not incorporate further methods to speed up the convergence process to have a predictable base line for the algorithms. Therefore, we accept the higher computational effort. For the proposed method, we choose $\beta=0.01$ and $\gamma=0.02$. These parameters were used in all simulations. The choice of these values is explained in detail in Sec. 3.

\section{C. System geometry}

As we want to investigate the performance of AIR for a state-of-the-art scanning modality, we base the simulations on the fan-beam geometry of the Somatom Definition Flash scanner (Siemens Healthcare). The scanner has a distance from the source to the center of rotation of $R_{\mathrm{F}}=595.0 \mathrm{~mm}$ and a distance from the detector to the center of rotation of $R_{\mathrm{D}}=490.6 \mathrm{~mm}$. In total $N=1160$ projections per rotation are acquired over an angular range of $360^{\circ}$. The detector con- sists of $M=736$ detector columns. A circular scan with a single $\mathrm{x}$-ray source was simulated.

\section{D. Phantom}

For all simulations, we assumed a monoenergetic x-ray spectrum. The mathematical definition of the phantom allows for an analytical calculation of the line integrals during rawdata simulation, which can be described mathematically as

$$
\bar{p}_{\mathrm{nm}}=-\ln \frac{\bar{I}_{\mathrm{nm}}}{I_{0}}=\int_{0}^{\infty} d \lambda f\left(\boldsymbol{s}_{n}+\lambda \boldsymbol{\Theta}_{\mathrm{nm}}\right) .
$$

Provided that $f$ is the ideal image (without noise), $\bar{I}_{\mathrm{nm}}$ is the ideal measured intensity (number of photons) for projection number $n \in\{1, \ldots, N\}$ and detector element $m \in\{1, \ldots, M\}$ without noise. $I_{0}$ is the unattenuated intensity, $\bar{p}_{\text {nm }}$ is the ideal projection value without noise. For rawdata simulation, we used Eq. (13) to create the ideal projection data. The points $\boldsymbol{s}_{n}$ are the source positions and $\boldsymbol{\Theta}_{\mathrm{nm}}$ are the unit vectors pointing from $s_{n}$ to the center points of the detector elements. From the analytical intensities $\bar{I}_{\mathrm{nm}}$, we generated Poisson-distributed noisy intensities $I_{\mathrm{nm}}$ (representing the measured intensities) with mean and variance $\bar{I}_{\mathrm{nm}}$. The constant value $I_{0}$ is the number of photons which a nonattenuated ray deposits per projection angle $n$ in each detector element $m$. In all simulations, $I_{0}$ was set to the same value $I_{0}=1.0 \times 10^{5}$, which results in approximately $30 \mathrm{HU}$ noise in the reconstructed FBP reference images, measured in a homogeneous region denoted with $\mathrm{D}$ in water-equivalent tissue (indicated with a circle in Fig. 1). Thus, we obtain the Poisson-distributed projection values as $p_{\mathrm{nm}}=-\ln \left(I_{\mathrm{nm}} / I_{0}\right)$. Figure 1 shows the ground truth of the resolution phantom (left) we used for the simulations and the analytical reference reconstruction (right) which is a FBP with a Ram-Lak kernel. ${ }^{29}$ To quantitatively and qualitatively investigate the algorithms, resolution bar patterns are used. The bar patterns have a resolution ranging from 4.2 to $14.5 \mathrm{LP} / \mathrm{cm}$, covering the total relevant resolution bandwidth. The high contrast resolution patterns have a CT-value of 1000 $\mathrm{HU}$, the resolution patterns with lower contrast are at $200 \mathrm{HU}$. The low contrast disks have 100, 50, and 25 HU. The background is water-equivalent. The diameter of the phantom is $160 \mathrm{~mm}$. The reconstructed field of view has a diameter of $200 \mathrm{~mm}$ comprising $N_{x} \times N_{y}=512 \times 512$ pixels resulting in a grid spacing of $\Delta x=\Delta y=0.39 \mathrm{~mm}$.

\section{E. Bilateral filter}

In this paper, we use the bilateral filter ${ }^{30,31}$ as an edgepreserving postreconstruction filter to generate basis images for the experiments in Sec. 3.A.4. Thus, we can introduce prior knowledge of smoothness with preserved sharpness into the reconstruction process. For this purpose, we define the bilateral filter in one dimension as

$$
\mathrm{B} f(x)=\frac{\int d t D(x, t) R_{f}(x, t) f(t)}{\int d t D(x, t) R_{f}(x, t)},
$$



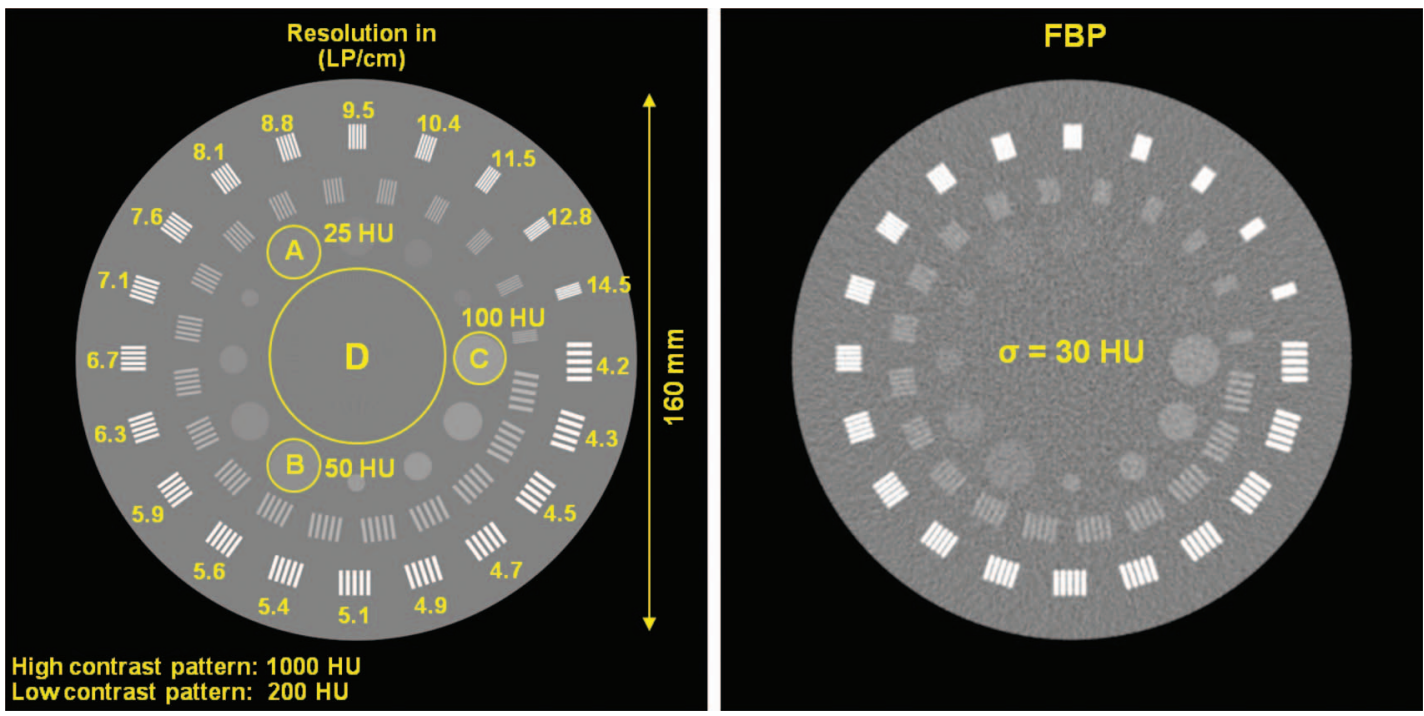

FIG. 1. Left: Ground truth of the phantom. The bar patterns have a resolution ranging from 4.2 to $14.5 \mathrm{LP} / \mathrm{cm}$. Noise was measured within the ROI D. Right: FBP reconstruction from noisy projections. $C=0 \mathrm{HU}, W=1000 \mathrm{HU}, \sigma=30 \mathrm{HU}$.

with B denoting the bilateral filtering operator and

$$
\begin{aligned}
& D(x, t)=e^{-\left(\frac{x-t}{\sigma_{x}}\right)^{2},} \\
& R_{f}(x, t)=e^{-\left(\frac{f(x)-f(t)}{\sigma_{f}}\right)^{2}} .
\end{aligned}
$$

Here, $f(x)$ is the unfiltered image and $\mathrm{B} f(x)$ is the filtered image. The bilateral filter consists of a domain part denoted with $D(x, t)$ and a range part denoted with $R_{f}(x, t)$. The domain part is a Gaussian distribution for smoothing controlled by the parameter $\sigma_{x}$ and the range part is a Gaussian distribution for edge-preservation controlled by the parameter $\sigma_{f}$. The greater the difference of the values $f(x)$ and $f(t)$ the smaller the weight $R_{f}(x, t)$ resulting in less contribution of this value in averaging and thus preserving edges. An example for bilateral filtered image can be seen in Fig. 2.

\section{F. Assessment of image quality}

For visual inspection, we show the results of the different algorithms. Noise was quantified as the standard deviation within the ROI D (see Fig. 1). We assess the spatial resolution in a quantitative approach as we measured the contrast of material to background in dependence on the size of the structures of linepairs with line profiles. To assess the resolution potential for the phantom results, we calculate a contrast factor $\mathrm{CF}$ as a function of the spatial resolution evaluated on the high contrast resolution bar patterns. To calculate the contrast, averaged line profiles are evaluated which cover $90 \%$ of the width of the bar patterns. The three inner local gray-value maxima $\operatorname{Max}(i)$ and minima $\operatorname{Min}(i)$ of the averaged line profile are evaluated and averaged. The $\mathrm{CF}$ is given by

$$
\mathrm{CF}=\frac{\overline{\operatorname{Max}(i)}-\overline{\operatorname{Min}(i)}}{\mathrm{E}-\mathrm{F}}
$$

where $\mathrm{F}$ is the ideal attenuation of the background $(0 \mathrm{HU})$, $\mathrm{E}$ the ideal attenuation of the bar patterns (1000 HU),
$\overline{\operatorname{Max}(i)}=\frac{1}{3} \sum_{i=1}^{3} \operatorname{Max}(i)$ the mean of the three inner maxima, and $\overline{\operatorname{Min}(i)}=\frac{1}{3} \sum_{i=1}^{3} \operatorname{Min}(i)$ the mean of three inner minima. This is done for each bar pattern that can be resolved in the reconstructions and the calculated CFs are plotted against the corresponding resolution in $\mathrm{LP} / \mathrm{cm}$ (contrast-resolution plots). For a quantitative assessment of the reconstruction quality, the normalized cross-correlation (NCC) between the reconstruction results and the ground truth is used. The NCC between a region $\Omega$ in the reconstructed image $f$ and a region in the ground truth image $g$ is given by

$$
\mathrm{NCC}=\frac{1}{|\Omega|} \sum_{x, y \in \Omega} \frac{(f(x, y)-\bar{f})(g(x, y)-\bar{g})}{\sigma_{f} \sigma_{g}},
$$

where $\bar{f}, \bar{g}$ are the mean values and $\sigma_{f}, \sigma_{g}$ the standard deviations over the image region $\Omega$. The number of pixels contained in $\Omega$ is $|\Omega|$. The NCC ranges from $[-1,1]$. A value of +1 means the images have a perfect positive relation. The closer the results are to +1 the closer is the reconstructed image to the ground truth. The region $\Omega$ for the NCC analysis are all values $f_{b}$ of the phantom exceeding $-500 \mathrm{HU}$ (i.e., air is not considered). For this region, we also calculate the root-mean-square deviation between the ground truth and the reconstruction results as a measure of image quality defined as

$$
\operatorname{RMSD}=\sqrt{\frac{1}{|\Omega|} \sum_{x, y \in \Omega}(f(x, y)-g(x, y))^{2}} .
$$

The smaller the value of the RMSD the closer is the reconstruction result to the ground truth. Furthermore, we calculate the contrast-to-noise ratio (CNR) of the low contrast disks A and $\mathrm{B}$ to the water equivalent background in the ROI D (see Fig. 1). The CNR is defined as 

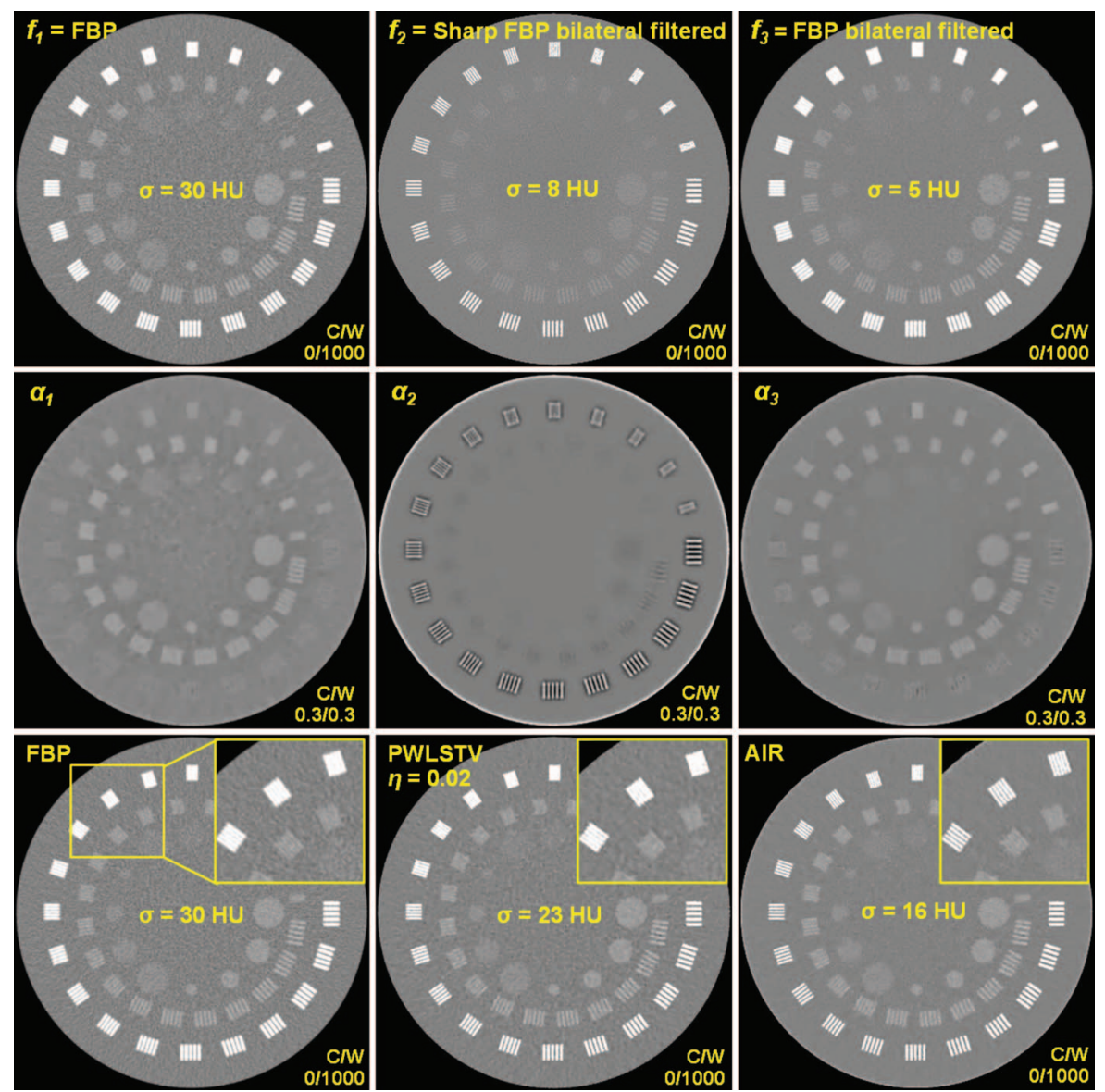

FIG. 2. Results of the experiments with three basis images.

$$
\operatorname{CNR}(\mathrm{X})=\frac{|\overline{\mathrm{X}}-\overline{\mathrm{D}}|}{\sqrt{\sigma_{\mathrm{X}}^{2}+\sigma_{\mathrm{D}}^{2}}},
$$

where $\overline{\mathrm{X}}$ is the mean value of the desired $\mathrm{ROI}(\mathrm{A}$ or $\mathrm{B})$ and $\overline{\mathrm{D}}$ is the mean value of the water background. The values $\sigma_{\mathrm{X}}$ and $\sigma_{\mathrm{D}}$ are the corresponding standard deviations.

\section{RESULTS AND DISCUSSION}

\section{A. Basic experiments}

\section{A.1. One basis image}

We start the analysis of the AIR algorithm by using only one basis image. We vary the trade-off parameter $\beta$ of AIR to demonstrate its influence on the weighting image $\alpha$. As basis image we use the reference FBP reconstruction. We use three different settings for the parameter $\beta \in\{0.0,0.01,1.0\}$. Figure 3 shows the simulation results. The top row shows the FBP reconstruction and the result of the WLS reconstruction (PWLS reconstructed with $\eta=0.0$, at convergence). Rows two to four show the results for the different settings for $\beta$. As we only use the FBP reconstruction as one basis image, the algorithm only reconstructs the $\alpha$-image $\alpha_{1}$. For $\beta=0.0$ no regularization is applied to the $\boldsymbol{\alpha}$-image. Thus, the reconstructed $\boldsymbol{\alpha}_{1}$ is noisy which is undesired (see Fig. 3, row two).
In column three of row two, one can see the difference image between the proposed method and the WLS reconstruction. The results are nearly identical. To find a good standard value for $\beta$, we did a parameter sweep to find a suitable value for $\beta$. We choose $\beta$ just large enough, so that the weighting images are smooth in homogeneous regions. This is achieved with $\beta$ $=0.01$ (see Fig. 3, row three). One can see that $\alpha_{1}$ is smooth in the homogeneous water background regions. Thus, the AIR result will have the same noise as the basis image FBP. As we do not want to introduce noise through the weighting images we use this value for $\beta$ in all further simulations in Secs. 3.A.2-3.A.4. As we do not regularize directly in the resulting image $f_{\text {AIR }}$, we are more flexible with the choice of $\beta$, as loss of details in the $\boldsymbol{\alpha}$-image will not necessarily lead to loss of details in the final result. For $\beta=0.01$, the difference image (row three column three) shows that the AIR result is mainly identical to the FBP reconstruction at homogeneous regions with the difference that the contrast of the resolution patterns is slightly improved, due to the weighting of the $\boldsymbol{\alpha}$-image. Further increasing the regularization strength to $\beta=1.0$ will lead to a homogeneous weight of 1.0 in $\alpha_{1}$ and thus the result will be identical to the basis image FBP. We can conclude that by choosing a proper value for $\beta$ no noise will be introduced by the weighted combination of the basis images. For simplicity, we use $\beta=0.01$ as the standard value for all further reconstructions. We are aware that the 


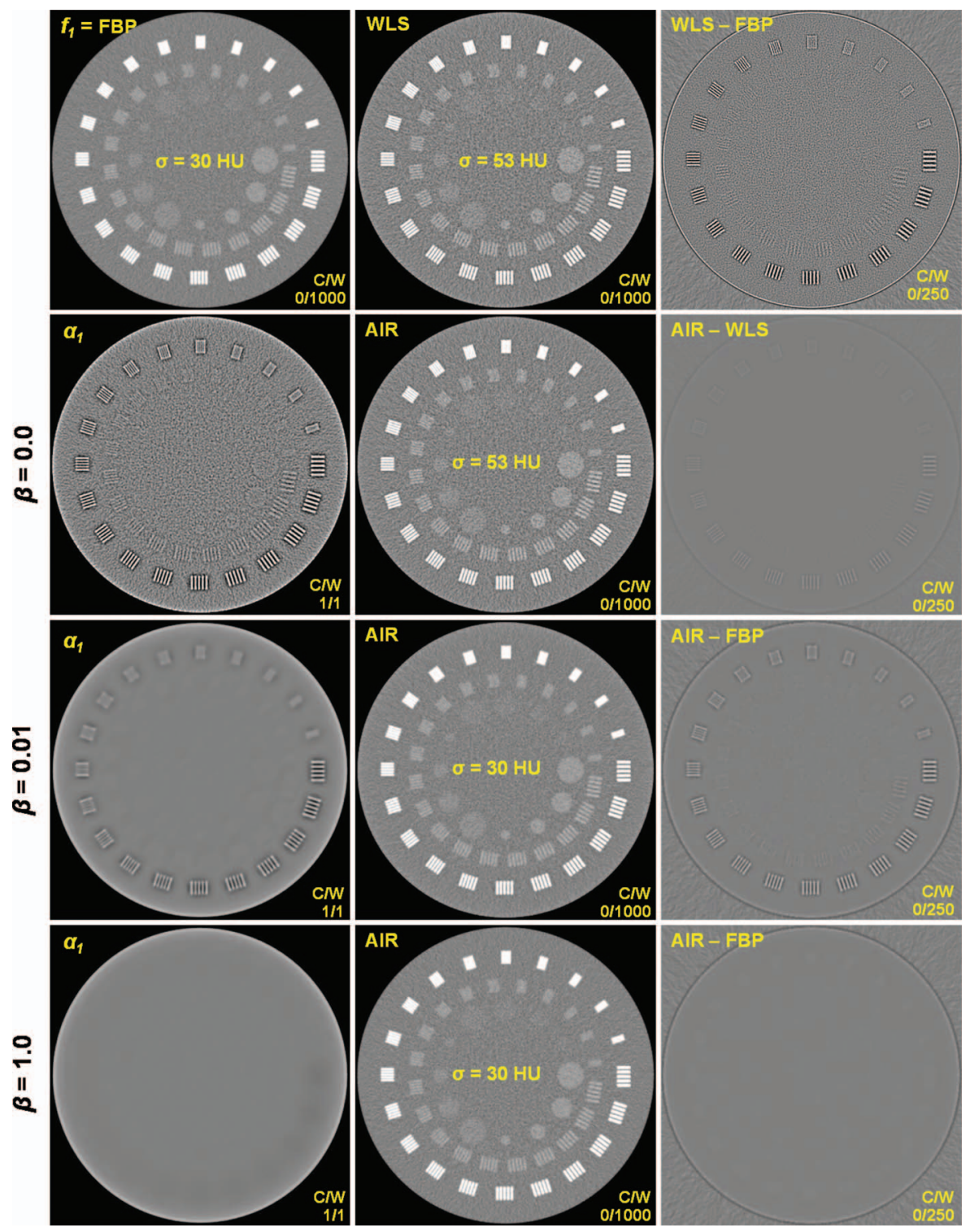

FIG. 3. Results of the experiments with one basis image.

weighting factor $\beta$ is, potentially, case-dependent. However, the focus of this work is to show the basic principle of the AIR algorithm and to demonstrate its functionality. We plan to investigate the influence of the reconstruction parameters on the final reconstruction result and to find optimal choices for these parameters in future research.

\section{A.2. Two basis images: Experiment 1}

This section and Sec. 3.A.3 are intended to demonstrate how the algorithm works in principle. To do so, we investigate the algorithm using two basis images. Figure 4 shows the results of this section. Basis image one again is the reference FBP reconstruction. By using FBP as one of the basis images one can be sure that all anatomical details are contained at least in one basis image. Furthermore, we incorporate smoothness as prior knowledge into basis image two. Basis image two is a very smooth version of the FBP reconstruction obtained by applying a Gaussian filter to the FBP reconstruction and adapting the FWHM of the filter so that the noise in the final image is approximately $1 \mathrm{HU}$ $(\mathrm{FWHM}=3.85 \mathrm{~mm})$. The result can be seen in row one of Fig. 4. Anatomical details are lost in $\boldsymbol{f}_{2}$ due to very strong nonedge-preserving filtering. The result of the AIR method is shown as well as the resulting reconstructed alpha images $\boldsymbol{\alpha}_{1}$ and $\boldsymbol{\alpha}_{2}$. One can see that the weights are high in $\boldsymbol{\alpha}_{1}$ in those regions of $\boldsymbol{f}_{1}$ where no details are visible in $\boldsymbol{f}_{2}$. This was expected, as the rawdata fidelity for $f_{1}$ in regions with anatomical detail is preferred over the blurry regions in $\boldsymbol{f}_{2}$. Thus, the detail information will be taken with from basis image $f_{1}$, i.e., higher weights in $\boldsymbol{\alpha}_{1}$, because this results in the lowest cost value for the sum of all three penalty functions. For a more detailed insight into why the weights are high in the regions in $\boldsymbol{\alpha}_{1}$ and low in the corresponding regions in $\boldsymbol{\alpha}_{2}$, please refer to Sec. 3.A.3. Due to the penalty term on the right hand side of Eq. (9) the default weighting for homogeneous regions 


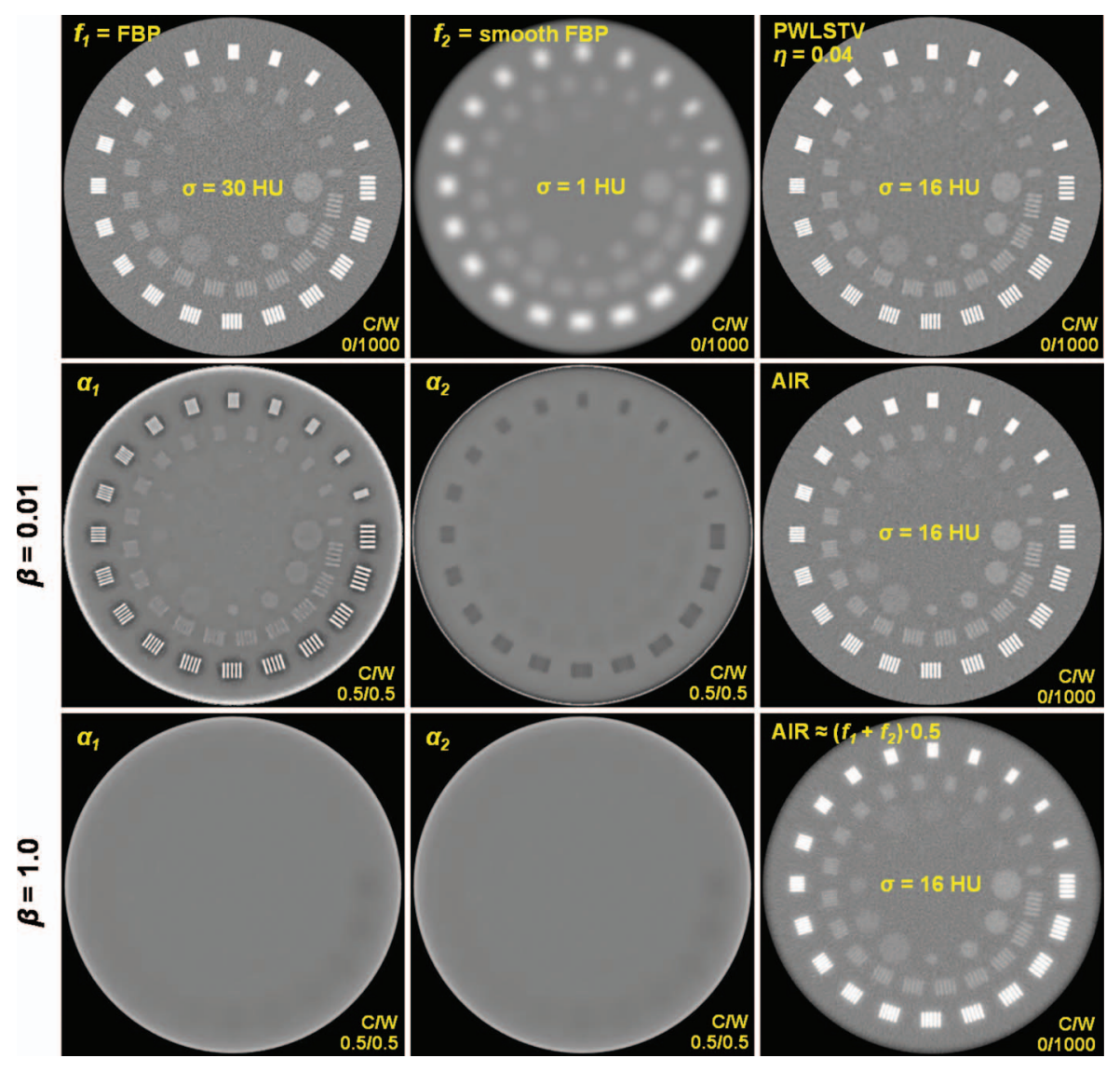

FIG. 4. Result of the first experiment with two basis images.

in $f_{1}$ and $f_{2}$, which do not strongly differ with respect to the rawdata fidelity, will be $d_{1}=d_{2}=1 / B=0.5$. This results in averaging the background content of the basis images. Thus, the noise in the final image will be averaged. To find a good value for parameter $\gamma$, a parameter sweep was performed. We chose $\gamma$ just large enough, so that the reconstructed weighting images $\boldsymbol{\alpha}_{b}$ default to $d_{b}$ in regions, which do not differ

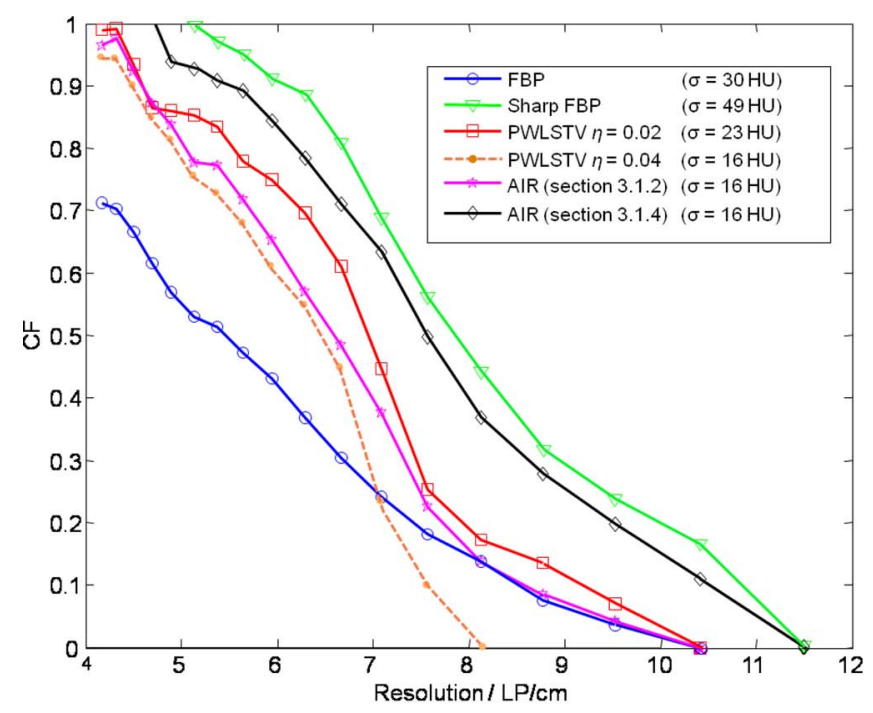

(a) with respect to their contribution to the rawdata fidelity. The anatomical detail will contribute with higher weights from the high resolution image. This is evaluated for the high contrast patterns with a contrast-resolution plot in Fig. 5(a). The CF of AIR is higher than the CF for FBP for patterns with a resolution lower than $8.0 \mathrm{LP} / \mathrm{cm}$, and almost identical to FBP from 8.0 LP/cm to the resolution limit. A comparison plot of line

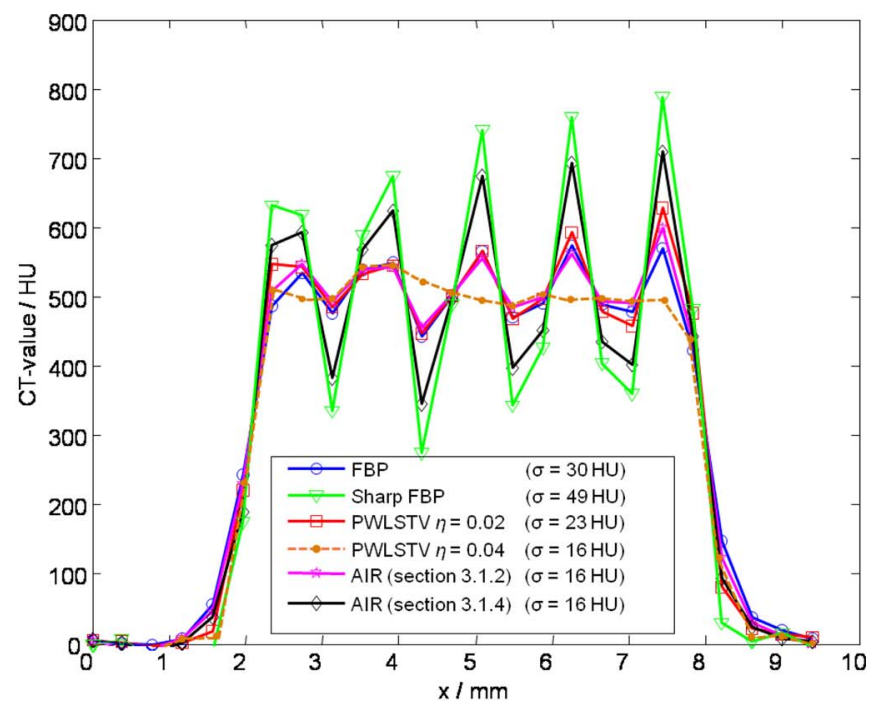

(b)

FIG. 5. (a) Contrast-resolution plots derived from high contrast patterns. (b) Line profiles through the resolution pattern with $8.1 \mathrm{LP} / \mathrm{cm}$. 
TABLE I. Quantitative assessment of image quality.

\begin{tabular}{lcccccc}
\hline \hline Algorithm & RMSD $\left[10^{-3}\right]$ & NCC & $\sigma[\mathrm{HU}]$ & CNR(A) & CNR(B) & Resolution limit (CF=0) $[\mathrm{LP} / \mathrm{cm}]$ \\
\hline FBP & 0.858 & 0.920 & 30 & 0.67 & 1.24 & 10.4 \\
Sharp FBP & 1.390 & 0.858 & 49 & 0.33 & 0.62 & 11.5 \\
PWLSTV $\eta=0.02$ & 0.674 & 0.953 & 23 & 0.88 & 1.65 & 10.4 \\
PWLSTV $\eta=0.04$ & 0.667 & 0.955 & 16 & 1.26 & 2.35 & 1.96 \\
AIR (Sec. 3.A.2) & 0.646 & 0.961 & 16 & 1.13 & 1.98 & 10.4 \\
AIR (Sec. 3.A.4) & 0.565 & 0.970 & 16 & 1.15 & 1.5 \\
\hline \hline
\end{tabular}

profiles through the $8.1 \mathrm{LP} / \mathrm{cm}$ resolution pattern can be seen in Fig. 5(b). Thus, the final reconstruction has the same high contrast resolution of $f_{1}$ but with approximately $50 \%$ noise. In row one, column three of Fig. 4 the PWLSTV reconstruction is shown where we use $\eta=0.04$, which results in approximately $16 \mathrm{HU}$ noise in ROI D. This is similar to the noise in AIR. Looking at the CF plot in Fig. 5(a) one can see the tradeoff between resolution and noise of the PWLSTV reconstruction. To achieve the low noise level of $16 \mathrm{HU}$, line patterns with a contrast in the range of the noise are oversmoothed. The proposed method can preserve the patterns due to the higher contribution from the sharp basis image in regions with high agreement with the rawdata fidelity [see Fig. 5(a)]. This shows the potential of the AIR algorithm. The AIR result also shows a higher agreement with the ground truth, measured with the NCC and the RMSD (see Table I). Row three of Fig. 4 shows the result when using $\beta=1$. . Now the TV penalty becomes the dominant part of the cost function. Both weighting images are completely smooth. The default contribution from both basis images is $d_{1}=d_{2}=1 / B=0.5$ due to the penalty term on the right hand side of Eq. (9). The final result will be simple averaging of the basis images. We can conclude from this experiment that AIR preserves anatomical details by choosing higher weights from the basis image with the most correct anatomical information. Noise reduc- tion is achieved by using at least one basis image which has low noise. A loss of anatomical information in this low noise basis image will be compensated for by the remaining basis images.

\section{A.3. Two basis images: Experiment 2}

To provide a more detailed insight into AIR, another experiment with two basis images was conducted. The images are shown in Fig. 6. We chose two extreme basis images which do not represent the use case but which are rather helpful to demonstrate how the algorithm works. We generated the two basis images by manipulating the FBP images. For basis image $f_{1}$, we strongly blurred the lower half circle of the FBP image and clipped the lower right quarter circle. On the one hand, basis image $\boldsymbol{f}_{1}$ contains regions where the detail information is correct, while on the other hand there are regions where the detail information is strongly degraded or completely missing. To generate basis image $\boldsymbol{f}_{2}$, we blurred the left half circle of the FBP image and we additionally included the DKFZ logo, which is the logo of our institution. This logo was added in image space with a CT-value of $500 \mathrm{HU}$. Neither the logo in $\boldsymbol{f}_{2}$, nor the lower right quadrant of $\boldsymbol{f}_{1}$, are contained in the projection data. They pose completely wrong and, potentially, misleading information in basis image $\boldsymbol{f}_{2}$.
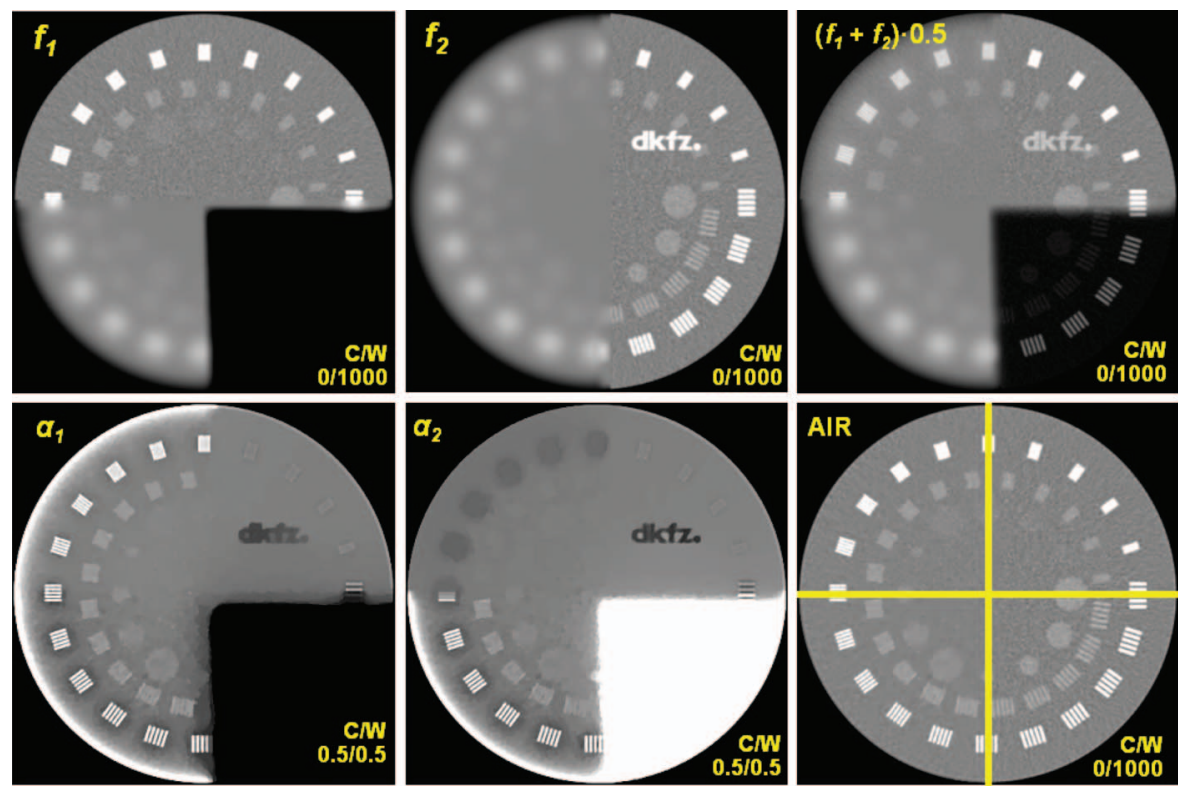

FIG. 6. Results of the second experiment with two basis images. 


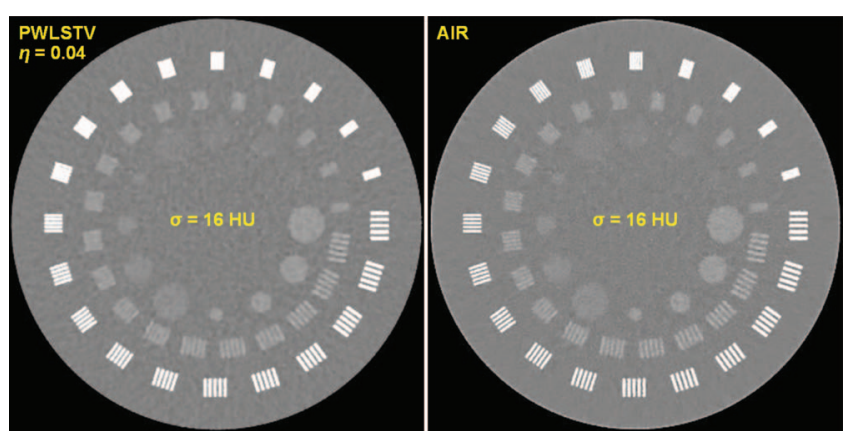

FIG. 7. Left: PWLSTV with $\eta=0.04$, resulting in a relative pixel noise of $16 \mathrm{HU}$, equal to the noise value in AIR (right).

These basis images were designed in order to discuss four cases in the corresponding reconstructed AIR result which are delimited by yellow lines in Fig. 6 .

Let us start with the upper right quadrant where both basis images have the same detail information (the one of the original FBP image). They are identical except for the DKFZ logo. When looking at the corresponding region in the reconstructed weighting images one can see that the weights are low in the regions where the resolution patterns are located in both weighting images, and they are nearly identical. This is because both basis images hold the same information and in both cases there is a high correlation with the projection data. When looking at the region of the DKFZ logo in the reconstructed weighting images one can see that both weighting images are low and almost equal in this particular region. Intuitively, one would probably expect that the weights in this region are high in the $\boldsymbol{\alpha}_{1}$ image and low in the $\boldsymbol{\alpha}_{2}$ image to compensate for the false information. This case would indeed lead to the same rawdata fidelity in the resulting image. However, the values for the remaining two penalty terms (the TV penalty and the mean contribution penalty) would be larger. Thus, the overall lowest cost can be obtained when the weights are equally low in both weighting images. In the AIR reconstruction, the DKFZ logo is not visible anymore which demonstrates that the AIR algorithm can compensate for false information in the basis images.

Now let us look at the lower right part of the AIR reconstruction. In this region, the information in basis image $f_{1}$ is completely missing. However, the corresponding region in basis image $f_{2}$ is correct and has a high agreement with the rawdata. Thus, $\boldsymbol{\alpha}_{2}$ is high and $\boldsymbol{\alpha}_{1}$ is low. The information will be transferred from $\boldsymbol{f}_{2}$ into the final AIR result.

In the lower left quadrant, the detail information in both basis images is completely missing. In this case, the algorithm tries to recover the detail information solely by the weights in $\boldsymbol{\alpha}_{1}$ and $\boldsymbol{\alpha}_{2}$. Here, one can again see that the weights are very similar in both reconstructed weighting images, because this results in the lowest cost for the TV and the mean penalty.

In the upper left quadrant, we have the same constellation as in Sec. 3.A.2 where $f_{1}$ corresponds to the FBP reconstruction while $\boldsymbol{f}_{2}$ is degraded by blurring. Consequently, the corresponding weights are high in $\boldsymbol{\alpha}_{1}$ and low in $\boldsymbol{\alpha}_{2}$ because this results in the lowest cost value for the sum of all three penalty functions. One would probably expect that the weights in the regions of the line patterns are about 1 in $\boldsymbol{\alpha}_{1}$ and about 0 in $\boldsymbol{\alpha}_{2}$. This could be expected when one only considers the rawdata fidelity penalty. However, this would increase the other two penalty terms which demand smoothness in the reconstructed weighting images, and the overall cost would not be at the minimum.

\section{A.4. Three basis images}

In this section, we present results of the AIR algorithm using three basis images. Moreover, we incorporate prior knowledge into the proposed method by applying the edgepreserving bilateral filter, described in Sec. 2.E, on some of the basis images. This section shows the performance of the algorithm regarding image quality. Figure 2 shows the results of the experiments in this section. Basis image $f_{1}$ again is the FBP reference reconstruction. The second basis image is a sharp FBP reconstruction which is postprocessed by the bilateral filter. The sharp FBP is obtained by convolving the sinogram with the kernel $(-1 / 3,5 / 3,-1 / 3)$ prior to reconstruction. The sharp FBP reconstruction performs well in the high contrast resolution patterns due to its high spatial resolution but performs bad in homogeneous regions due to the high noise. We want to transfer the wanted information of the sharp FBP reconstruction into our final result. We can perform edge-preserving bilateral filtering ${ }^{30}$ to reduce background noise without degrading the high contrast structures. To do so we use a high value for the domain filter $\sigma_{f}=500$ $\mathrm{HU}$, and a large range of $\sigma_{x}=4 \mathrm{~mm}$ for strong noise suppression and apply it to the sharp FBP reconstruction. The resulting basis image $f_{2}$ can be seen in Fig. 2. One can see that the high contrast structures are preserved but the low contrast structures are degraded leading to loss of anatomical information in $\boldsymbol{f}_{2}$. However, this poses no disadvantage when at least one basis image still contains this anatomical information. This is an advantage over standard regularization approaches, where one has to adapt filter parameters carefully. In the AIR algorithm, we can choose extreme filter settings for the various basis images to achieve a strong noise suppression. To generate basis image $f_{3}$, we apply the bilateral filter on the standard FBP reconstruction with a similar setting as before. The same range of $\sigma_{x}=4.0 \mathrm{~mm}$ is used, to achieve high noise suppression, but a smaller domain filter of $\sigma_{f}=100$ $\mathrm{HU}$ is used. The resulting basis image $\boldsymbol{f}_{3}$ has low noise, similar to the basis image $\boldsymbol{f}_{2}$ in Sec. 3.A.2 with the difference that most of the low contrast anatomical information is preserved through the edge-preserving bilateral filter. The result of AIR can be seen in Fig. 2. One can see in row three of Fig. 2 that the AIR method has the best high contrast resolution. Low contrast structures are preserved as well. The results of the quantitative comparison are summarized in Table I and Fig. 5. Figure 5(a) shows the contrast-resolution plots for the compared algorithms. One can see that AIR preserves the resolution of the sharp FBP reconstruction all the way to the resolution limit. At the same time, it sustains the lowest noise value. Table I shows the results for the CNR analysis and the agreement of the compared algorithms with the ground truth. AIR 

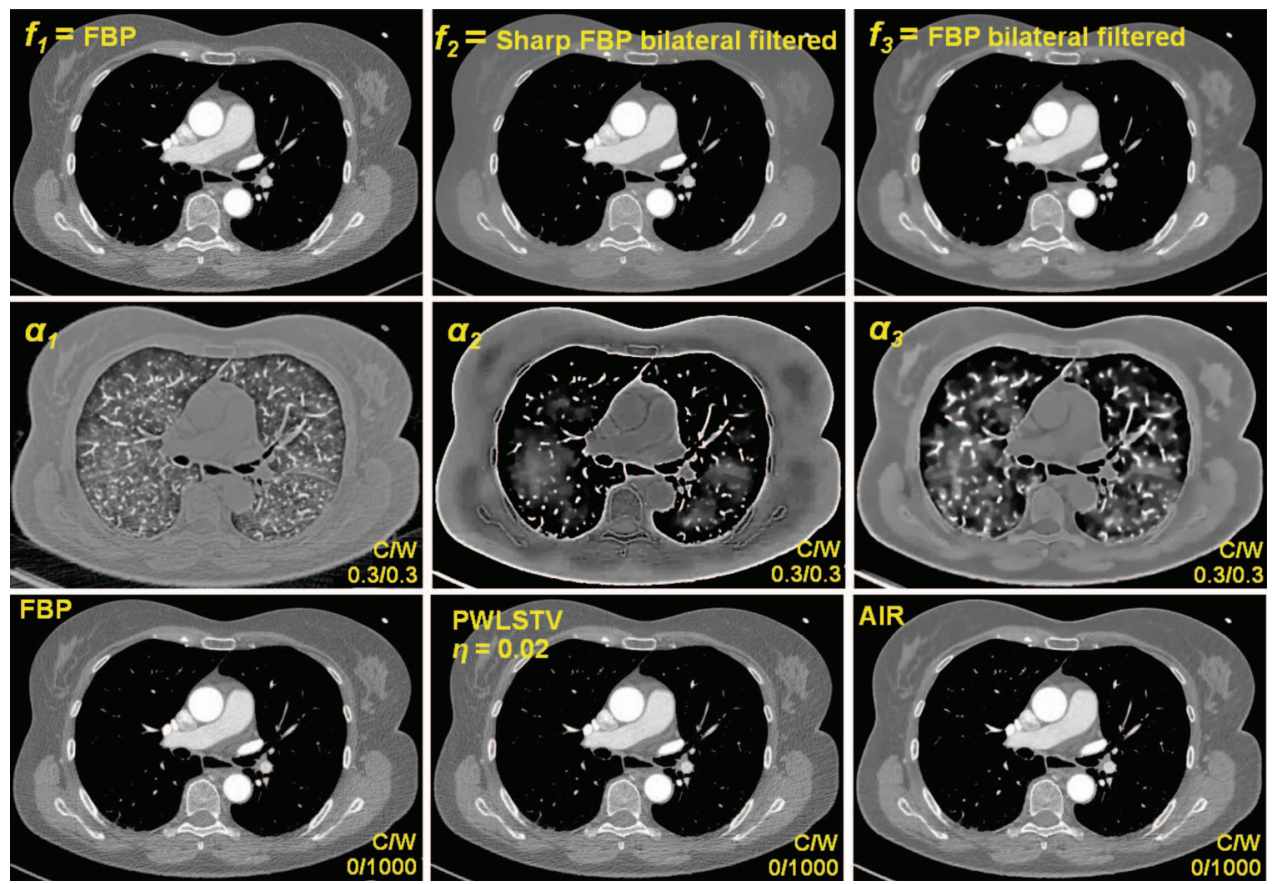

FIG. 8. Results of the patient data at constant zoom factor. The figure shows the FBP, the PWLSTV, and AIR with the corresponding basis images and weighting images.
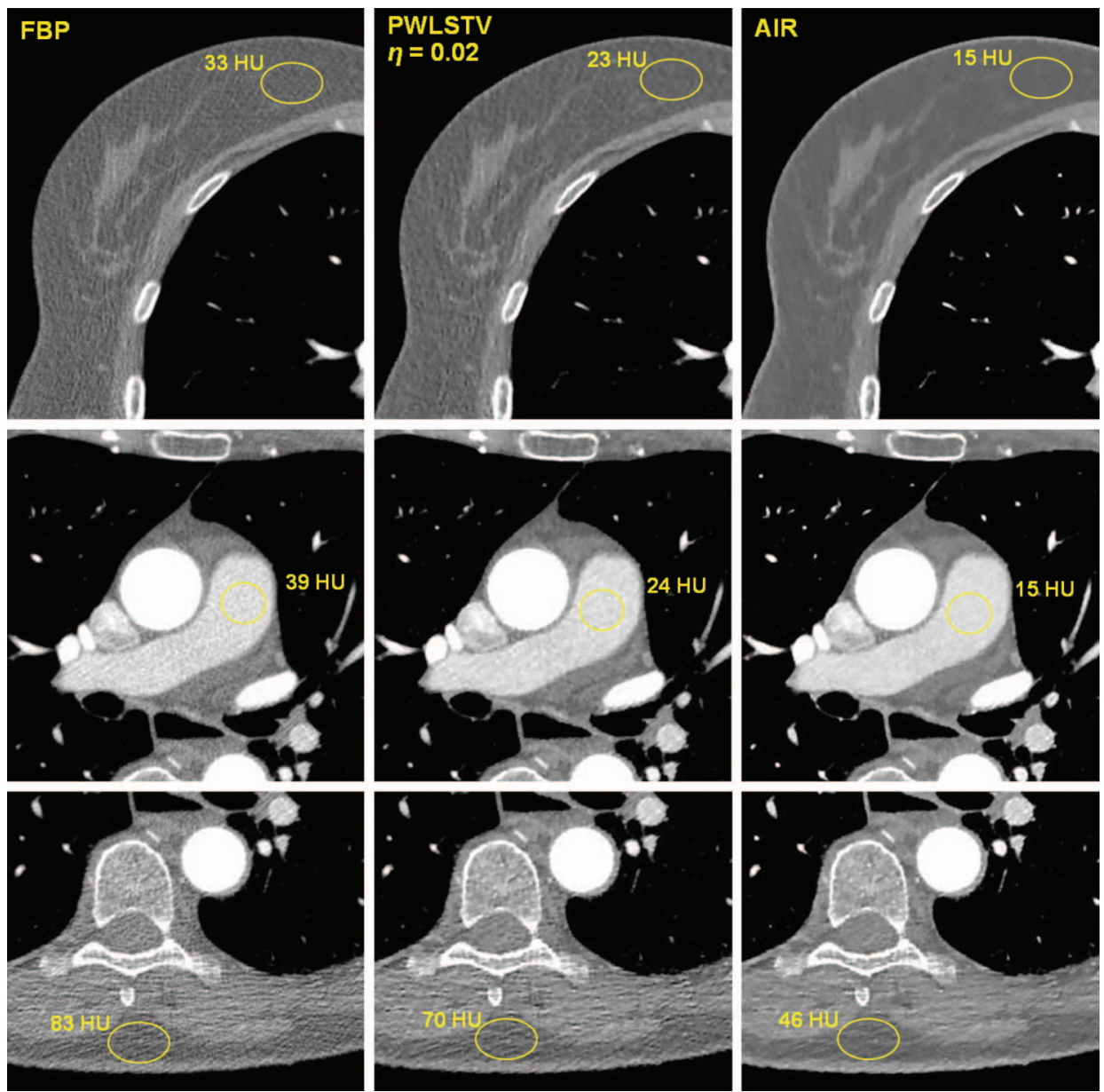

FIG. 9. Results of the patient data at constant zoom factor. The FBP result is compared to PWLSTV with $\eta=0.02$ and with AIR. The windowing is $C=0$ HU, $W=1000 \mathrm{HU}$. 
outperforms the remaining algorithms. Only the PWLSTV reconstruction with $\eta=0.04$ performs better referring to $\mathrm{CNR}(\mathrm{A})$ and $\mathrm{CNR}(\mathrm{B})$ with the downside of oversmoothed high and low contrast resolution patterns. Figure 7 shows the result of the PWLSTV with $\eta=0.04$ and AIR enlarged. The parameter $\eta$ was chosen so that the resulting relative pixel noise is the same as in AIR. Here, one can clearly see the trade-off between low noise and resolution. With higher regularization parameters $\eta$ the relative pixel noise decreases and at the same time anatomical details, which have a contrast in the order of magnitude of the noise, will disappear, which can be seen in the high contrast and low contrast resolution patterns of the PWLSTV in Fig. 7. AIR, however, can manage to maintain the detail information at the same noise level, thus improving the trade-off between noise and resolution. We can conclude, that the AIR algorithm appears to successfully maintain anatomical information from the basis images best representing the ground truth by taking the rawdata fidelity as underlying constraint. Finding optimal choices for the basis images will be the object of future research.

\section{B. Patient data}

To confirm the results of the phantom study, we used patient data acquired with a Somatom Definition Flash scanner. A sequence scan with 1160 projections at $100 \mathrm{kV}$ was acquired. The scanner has 736 detector columns and 64 active detector rows. We used the data of the two center rows for a $2 \mathrm{D}$ reconstruction. The reconstructed field of view has a diameter of $500 \mathrm{~mm}$ comprising $N_{x} \times N_{y}=1024 \times 1024$ pixels resulting in a grid spacing of $\Delta x=\Delta y=0.49 \mathrm{~mm}$. Figure 8 shows the basis images and the corresponding reconstructed weighting images. The same settings for $\beta=0.01, \gamma=0.02$ and the bilateral filter for the basis images $f_{2}$ and $f_{3}$ are used as in Sec. 3.A.4. Figure 9 shows the results of the methods compared (FBP, PWLSTV, AIR) enlarged for better visual inspection. Figure 10 shows the complete transversal slice of

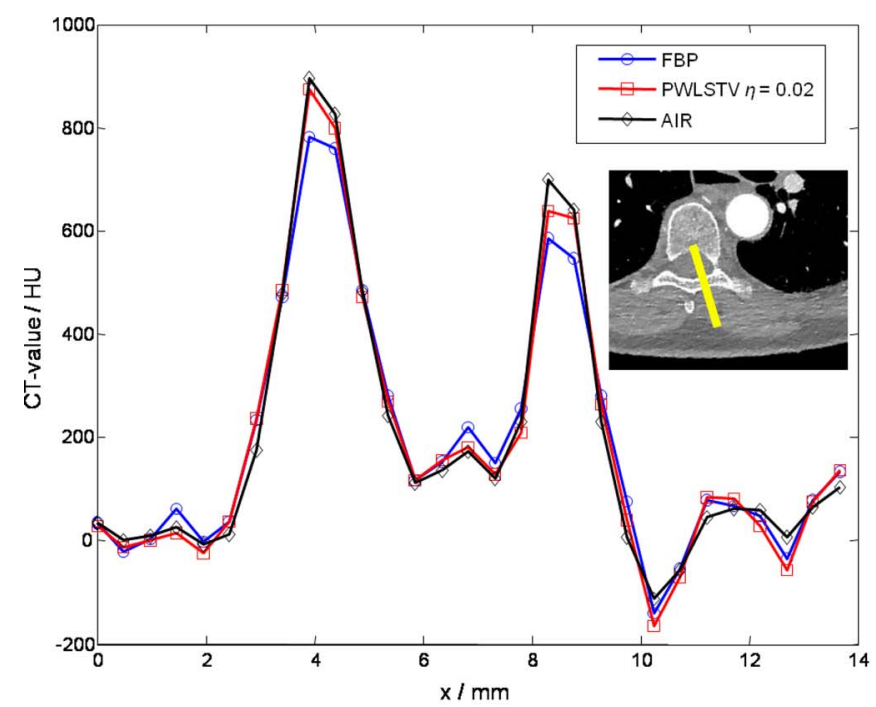

(a)

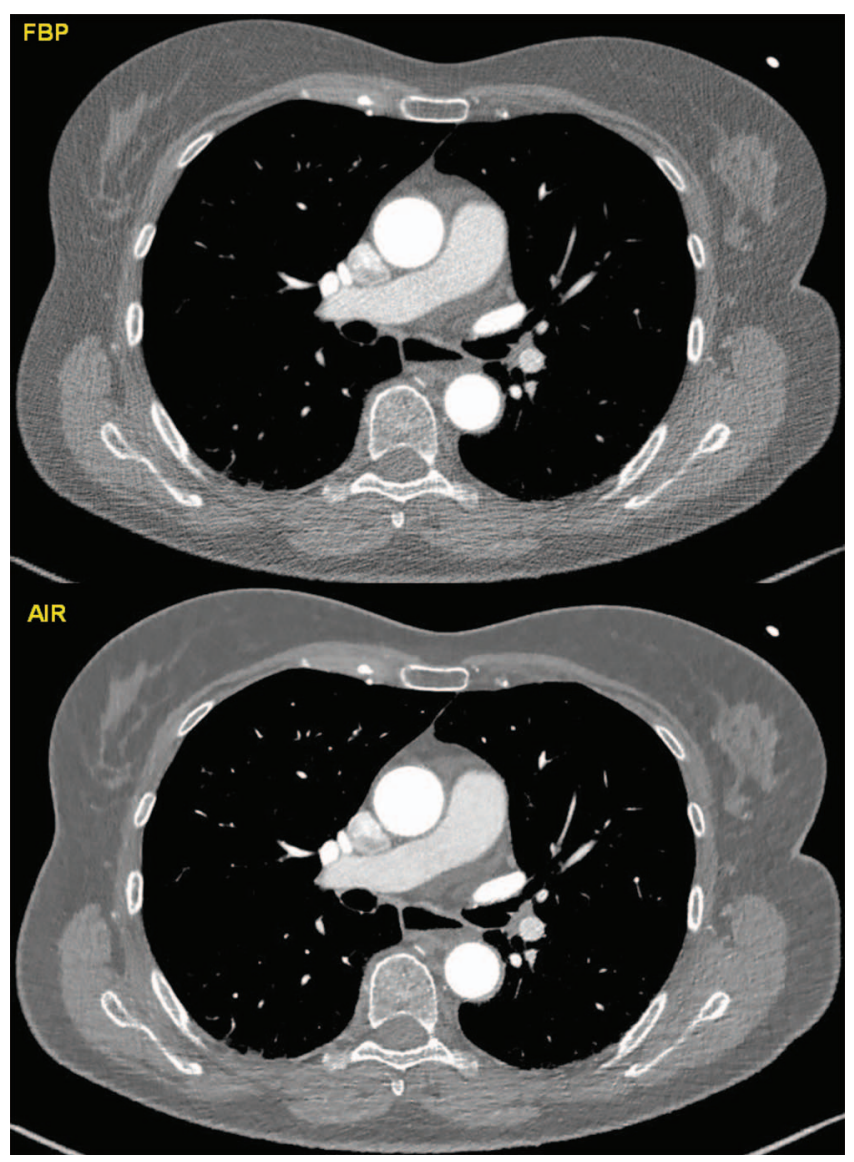

FIG. 10. Results of the patient data. Comparison of the proposed method with the FBP result. The windowing is $C=0 \mathrm{HU}, W=1000 \mathrm{HU}$.

the patient reconstructed with FBP and with AIR. The patient data results indicate that the AIR algorithm is, potentially, capable of achieving noise reduction values in the range of $50 \%$, compared to the FBP reconstruction, without altering the anatomical information. Figure 11 shows line profiles

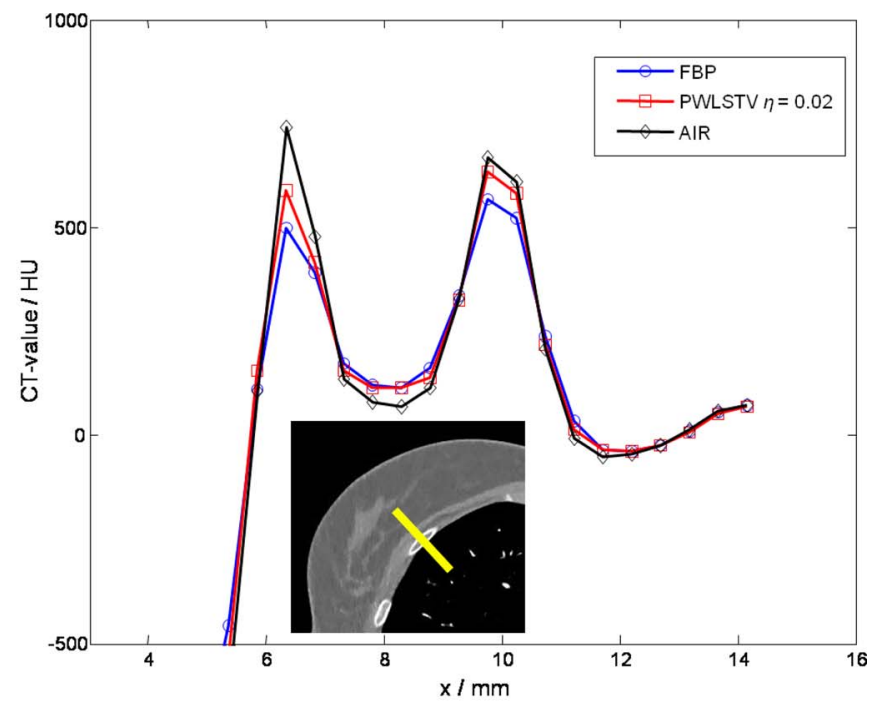

(b)

FIG. 11. Line profiles through (a) the vertebra, (b) the ridge. The locations of the line profiles are indicated with the lines. 
which are drawn through the vertebra and the ridge. One can see that AIR achieves the highest contrast, at the high contrast structures.

\section{CONCLUSION AND OUTLOOK}

In this work, we present a new method to incorporate prior knowledge into iterative reconstruction by using basis images which are combined by reconstructing voxel-specific weighting coefficients. Our method is called AIR. We were able to demonstrate in a phantom study, that the AIR algorithm is capable of reducing the relative pixel noise by up to $50 \%$ and at the same time maintaining the anatomical information given an adequate choice for the basis images. Any kind of filtering or regularization can be used to generate the basis images, thus making the method very flexible. The applicability of the proposed method on patient data was also demonstrated for patient data, measured with a Somatom Definition Flash scanner (Siemens Healthcare, Forchheim, Germany).

As one is very flexible with the choice of basis images future research may be concerned with finding optimal choices for basis images and with finding optimal choices for the values $d_{b}$, which control the default contribution of each basis image to the voxel-specific weighted linear combination. One example of a possible special application could be cardiac CT. Here AIR can, potentially, be used to reduce blooming artifacts and to optimize dose usage by using one basis image with high spatial and temporal resolution for reduced blooming artifacts and one basis image with lower spatial and temporal resolution which contains all redundant data obtained during the cardiac scan.

Another direction of future investigation could be a slight generalization of the cost function

$$
\begin{aligned}
C(\boldsymbol{\alpha})= & \left\|\boldsymbol{X} \cdot\left(\sum_{b=1}^{B} \boldsymbol{\alpha}_{b} \circ \boldsymbol{f}_{b}\right)-\boldsymbol{p}\right\|_{\boldsymbol{W}}^{2}+\beta \sum_{b=1}^{B} P_{b}\left(\boldsymbol{\alpha}_{b}\right) \\
& +\gamma \sum_{b=1}^{B}\left\|\boldsymbol{\alpha}_{b}-\boldsymbol{d}_{b}\right\|_{2}^{2}+\delta P\left(\sum_{b}^{B} \boldsymbol{\alpha}_{b} \circ \boldsymbol{f}_{b}\right) .
\end{aligned}
$$

This includes more general penalties $P_{b}$ for each alpha image, and it includes an additional penalty term $P$ that regularizes the final image $\boldsymbol{f}=\sum_{b=1}^{B} \boldsymbol{\alpha}_{b} \circ \boldsymbol{f}_{b}$. In this paper, $P_{b}=\mathrm{TV}$ and $P=0$. Last but not least one should focus on the computational complexity of AIR in order to reduce its computation time. Using ordered subsets may probably increase the convergence rate by one or two orders of magnitude.

Summarizing, AIR is a new type of iterative CT image reconstruction algorithm that appears to be highly flexible. Its potential to reduce image noise while preserving spatial resolution was demonstrated in this paper. AIR is able to automatically select desired properties from the basis images while it simultaneously prevents false information to propagate to the final image. This may be an advantage over conventional types of iterative image reconstruction algorithms. Future in- vestigations may reveal additional interesting applications for AIR.

\section{ACKNOWLEDGMENTS}

This work was funded by the Deutsche Forschungsgemeinschaft (DFG) under Grant No. KA 1678/3-1. Parts of the reconstruction software were provided by RayConStruct ${ }^{\circledR}$ GmbH, Nürnberg, Germany.

a) Author to whom correspondence should be addressed. Electronic mail: marc.kachelriess@dkfz-heidelberg; Telephone: +49 (6221) 423067; Fax: +49 (6621) 422585.

${ }^{1}$ M. Kachelrieß, "Iterative reconstruction techniques: What do they mean for cardiac CT?," Cardiovasc. Imaging Rep. 6, 268-281 (2013).

${ }^{2}$ S. Steckmann and M. Kachelrieß, "Blooming artifact reduction for cardiac CT," Nuclear Science Symposium Conference Record (NSS/MIC) (IEEE, Knoxville, TN, 2010), pp. 2030-2035.

${ }^{3}$ C. Hofmann and M. Kachelrieß, "Removing blooming artifacts with binarized deconvolution in cardiac CT," Proc. SPIE 9033, (2014).

${ }^{4}$ S. Do, W. Karl, Z. Liang, M. Kalra, T. Brady, and H. Pien, "A decomposition-based CT reconstruction formulation for reducing blooming artifacts," Phys. Med. Biol. 56(72), 7109-7125 (2011).

${ }^{5}$ Z. Liang, S. Do, U. Hoffmann, T. Brady, and H. Pien, "Calcium deblooming in coronary CT image," in Proceedings of the 7th IEEE International Conference on Bioinformatics and Bioengineering, Boston, MA, October 2007 (IEEE, 2007), pp. 257-262.

${ }^{6}$ J. Leipsic, T. LaBounty, B. Heilbron, J. Min, J. Mancini, F. Lin, C. Taylor, A. Dunning, and J. Earls, "Estimated radiation dose reduction using adaptive statistical iterative reconstruction in coronary CT angiography: The ERASIR study," Am. J. Roentgenol. 195(3), 655-660 (2010).

${ }^{7}$ H. Scheffel, P. Stolzmann, C. Schlett, L. Engel, G. Major, M. Karolyi, S. Do, P. Maurovich, and U. Hoffmann, "Coronary artery plaques: Cardiac CT with model-based and adaptive-statistical iterative reconstruction technique,” Eur. J. Radiol. 81, 363-369 (2012).

${ }^{8}$ M. Bittencourt, B. Schmidt, M. Seltmann, G. Muschiol, D. Ropers, W. Daniel, and S. Achenbach, "Iterative reconstruction in image space (IRIS) in cardiac computed tomography: Initial experience," Int. J. Cardiovasc. Imaging 27, 1081-1087 (2011).

${ }^{9}$ U. Ebersberger, F. Tricarico, U. Schoepf, P. Blanke, J. Spears, G. Rowe, W. Halligan, T. Hensler, F. Bamberg, A. Leber, E. Hoffman, and P. Apfalter, "CT evaluation of coronary artery stents with iterative image reconstruction: Improvements in image quality and potential for radiation dose reduction," Eur. Radiol. 23(1), 125-132 (2013).

${ }^{10}$ W. Hosch, W. Stiller, D. Mueller, G. Gitsioudis, J. Welzel, M. Dadrich, S. Buss, E. Giannitsis, H. Kauczor, H. Katus, and G. Korosoglou, "Reduction of radiation exposure and improvement of image quality with BMI-adapted prospective cardiac computed tomography and iterative reconstruction," Eur. Radiol. 81, 3568-3576 (2012).

${ }^{11}$ R. Nelson, S. Feuerlein, and D. Boll, "New iterative reconstruction techniques for cardiovascular computed tomography: How do they work, and what are the advantages and disadvantages?," J. Cardiovasc. Comput. Tomogr. 5, 286-292 (2011).

${ }^{12}$ M. Willemink, P. Jong, T. Leiner, L. Heer, R. Nievelstein, R. Budde, and A. Schilham, "Iterative reconstruction techniques for computed tomography Part 1: Technical principles," Eur. Radiol. 23(6), 1623-1631 (2013).

${ }^{13}$ J. Leipsic, B. Heilbron, and C. Hague, "Iterative reconstruction for coronary CT angiography: Finding its way," Int. J. Cardiovasc. Imaging 28, 613-620 (2012).

${ }^{14}$ A. Ziegler, T. Koehler, and R. Proksa, "Noise and resolution in images reconstructed with FBP and OSC algorithms for CT," Med. Phys. 34(2), 585-598 (2007).

${ }^{15}$ M. Kachelrieß, T. Berkus, and W. A. Kalender, "Quality of statistical reconstruction in medical CT," IEEE Medical Imaging Conference Program (IEEE, 2003), p. 153.

${ }^{16}$ M. Persson, D. Bone, and H. Elmqvist, "Total variation norm for threedimensional iterative reconstruction in limited view angle tomography," Phys. Med. Biol. 46(3), 853-866 (2001).

${ }^{17}$ J.-B. Thiabault, K. Sauer, C. Bouman, and J. Hsieh, "High quality iterative reconstruction for multi-slice helical CT," in Proceedings of the 
International Conference on Fully 3D Reconstruction in Radiology and Nuclear Medicine, Saint Malo, July 2003.

${ }^{18} \mathrm{H}$. Bruder, R. Raupach, and J. Sunnegardh, "Adaptive iterative reconstruction,” Proc. SPIE 7961, 1-12 (2011).

${ }^{19}$ L. Ouyang, T. Solberg, and J. Wang, "Effects of the penalty on the penalized weighted least-squares image reconstruction for low-dose CBCT," Phys. Med. Biol. 56(17), 5535-5552 (2011).

${ }^{20}$ J.-B. Thibault, K. D. Sauer, C. A. Bouman, and J. Hsieh, "A threedimensional statistical approach to improved image quality for multislice helical CT," Med. Phys. 34(11), 4526-4544 (2007).

${ }^{21}$ W. Chlewicki, F. Hermansen, and S. Hansen, "Noise reduction and convergence of Bayesian algorithms with blobs based on the Huber function and median root prior," Phys. Med. Biol. 49, 4717-4730 (2004).

${ }^{22} \mathrm{D}$. Yu and J. Fessler, "Iterative total-variation reconstruction versus weighted filtered-backprojection reconstruction with edge-preserving filtering," IEEE Trans. Med. Imaging 21(2), 159-173 (2002).

${ }^{23}$ G. Zeng, Y. Li, and A. Zamyatin, "Edge-preserving tomographic reconstruction with nonlocal regularization," Phys. Med. Biol. 58, 3413-3431 (2013).

${ }^{24}$ J. Wang, T. Li, and L. Xing, "Iterative image reconstruction for CBCT using edge-preserving prior,” Med. Phys. 36(1), 252-260 (2009).
${ }^{25}$ C. Hofmann, M. Knaup, and M. Kachelrieß, "A new approach to regularized iterative CT image reconstruction,” Proc. SPIE 9033, (2014).

${ }^{26} \mathrm{C}$. Bouman and K. Sauer, "A unified approach to statistical tomography using coordinate descent optimization," Trans. Image Process. 5(3), 480492 (1996).

${ }^{27} \mathrm{P}$. M. Joseph, "An improved algorithm for reprojecting rays through pixel images," IEEE Trans. Med. Imaging 1(3), 192-196 (1982).

${ }^{28}$ P. Lauzier, J. Tang, and G. Chen, "Prior image constrained compressed sensing: Implementation and performance evaluation,” Med. Phys. 39(1), 66-80 (2012).

${ }^{29}$ G. Ramachandran and A. Lakshminarayanan, "Three-dimensional reconstruction from radiographs and electron micrographs: Application of convolution instead of Fourier transforms," Proc. Natl. Acad. Sci. U.S.A. 68(9), 2236-2240 (1971).

${ }^{30}$ C. Tomasi and R. Manduchi, "Bilateral filtering for gray and color images," in Proceedings of the 6th International Conference on Computer Vision, Bombay, 1998 (IEEE, 1998), pp. 839-846.

${ }^{31} \mathrm{~W}$. Xu and K. Mueller, "A performance-driven study of regularization methods for GPU-accelerated iterative CT," in Proceedings of the 2nd High Performance Workshop, Beijing China, 2009 (Fully 3D conference proceedings, Beijing, China, 2009), pp. 1-4. 